\title{
Special Issue: ACM SIGMETRICS 2014
}

\author{
Marc Lelarge $^{1}$ - Florian Simatos ${ }^{2}$
}

Published online: 14 September 2015

(C) Springer Science+Business Media New York 2015

The five papers in this special issue are based on a selection of top theoretical papers from the ACM SIGMETRICS International Conference on Measurement and Modeling of Computer Systems, which took place at Austin, Texas, in June 2014. As the premier conference on the measurement and modeling of computer systems, the technical program featured papers that covered both theory and applications from a wide variety of areas, including algorithms, communication networks, dynamics and control, optimization, performance analysis, resource allocation and scheduling, and stochastic modeling, among others. The program consisted of 40 papers selected from more than 237 international submissions by a technical program committee of well-established researchers from all over the world. We seek with this special issue to highlight some of the recent theoretical work on the mathematical analysis and modeling of computer systems.

The paper of Larranaga, Ayesta, and Verloop deals with a resource allocation problem in a multi-class server with convex holding costs and user impatience under the average cost criterion. Although the optimal policy has a complex dependency on all the input parameters and state information, the authors derive simple index policies obtained by solving a relaxed version of the optimal stochastic control problem and combining results from restless multi-armed bandits and queueing theory.

Florian Simatos

florian.simatos@isae.fr

Marc Lelarge

marc.lelarge@ens.fr

1 INRIA-ENS, 23, Avenue d'Italie, 75013 Paris, France

2 Département DISC, ISAE-SUPAERO, 10 Avenue Edouard Belin, BP 54032,

31055 Toulouse Cedex 4, France 
The paper of Jelenković and Skiani provides a better understanding of instabilities caused by retransmissions by showing that processor sharing-based scheduling can induce complete instability with zero throughput in the presence of retransmissions, regardless of how low the traffic load may be.

The paper of Meyfroyt, Borst, Boxma, and Denteneer shows that the broadcasting process of a network using Trickle can be modeled by a Markov chain. By properly choosing the length of a listen-only period, the authors show how to greatly increase the speed at which the algorithm can propagate updates, while still controlling the number of transmissions.

The paper of Moharir, Ghaderi, Sanghavi, and Shakkottai looks at content placement in the high-dimensional regime, i.e., when the number of contents is of the same order as the number of servers, and proposes simple adaptive content placement schemes.

The paper of Walton obtained the Best Paper Award of the conference. It considers a generalized class of switch policies and shows that these share the same maximum stability properties as the MaxWeight class of policies. For the Proportional Scheduler, these stability results are extended to multihop networks with fixed routing.

We are grateful to the authors for amending and expanding their conference papers into journal versions tailored to the Queueing Systems community. The papers have gone through a regular Queueing Systems review cycle, and we are indebted to the anonymous referees not only for the quality of their work, but also for their assistance in working with our tight timeline. 